\title{
Quasi-ballistic Electronic Thermal Conduction in Metal Inverse Opals
}

\author{
Michael T. Barako ${ }^{1}$, Aditya Sood ${ }^{1,2}$, Chi Zhang ${ }^{1}$, Junjie Wang ${ }^{3}$, Takashi Kodama ${ }^{1}$, Mehdi \\ Asheghi ${ }^{1}$, Xiaolin Zheng ${ }^{1}$, Paul V. Braun ${ }^{3}$, and Kenneth E. Goodson ${ }^{1, *}$
}

\footnotetext{
${ }^{1}$ Department of Mechanical Engineering, Stanford University, Stanford, CA 94305

${ }^{2}$ Department of Materials Science and Engineering, Stanford University, Stanford, CA 94305

${ }^{3}$ Department of Materials Science and Engineering and Frederick Seitz Materials Research Laboratory, University of Illinois at Urbana-Champaign, Urbana, IL 61801

*Corresponding Author: goodson@ @stanford.edu
}

The following sections are included in the Supporting Information:

Section 1. Additional Details of Inverse Opal Synthesis

Section 2. Material Characterization of Inverse Opals

Section 3. $3 \omega$ Device Characterization, Sensitivity Analysis, and Uncertainty Analysis

Section 4. Additional Details of Conduction Model

Section 5. Electrical Conductivity Measurement

Section 6. Tradeoff between Surface Area-to-Volume Ratio and Thermal Conductivity Section 7. Supporting Information References 


\section{Section 1. Additional Details of Inverse Opal Synthesis}

For inverse opal synthesis onto microfabricated substrates, the Ti/Au metal seed layer is evaporated through a $3 \mathrm{~mm}$-diameter circular shadow mask onto the center of the device such that the exposed window over the buried working electrode forms electrical continuity between the contact pad and the seed layer. After functionalizing the seed layer surface to be hydrophilic, a hydrophobic fingernail lacquer is painted over the thermal measurement contact pads to prevent opal formation. The opal is formed on the seed layer, and a razor blade is used to cleave and remove the opal from the working electrode contact prior to annealing. In order to wet the opal with the electrolyte without damaging the crystal, a $3 \mu \mathrm{L}$ drop of isopropyl alcohol (IPA) is placed at the edge of the opal and allowed to wick laterally into the template. For nickel, after the entire opal is visibly wet with IPA the substrate is immediately placed in the electrolyte bath. For copper, a $3 \mu \mathrm{L}$ drop of the electrolyte is first placed on the opposite edge of the template; the IPA drop then wicks across the opal and mixes with the electrolyte drop. The substrate is then immersed in the electrolyte bath. After metal deposition, tetrahydrofuran simultaneously dissolves the polystyrene spheres, the fingernail lacquer, the epoxy, and the colloidal silver.

(a)

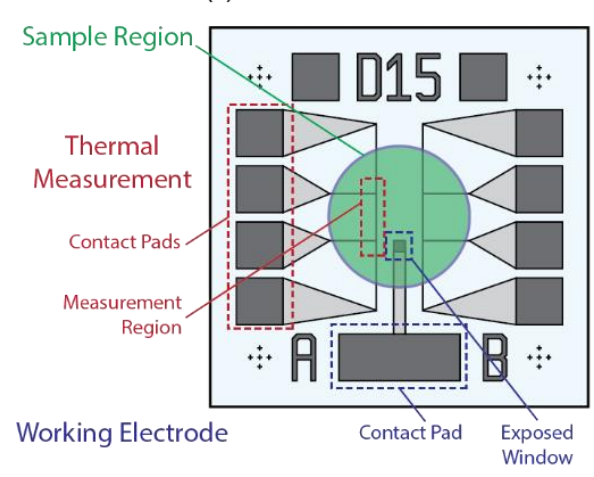

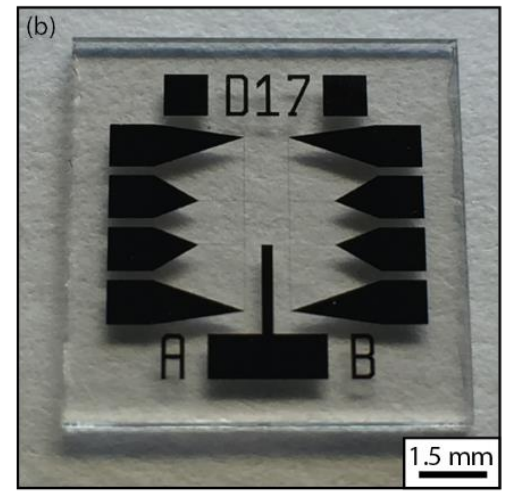

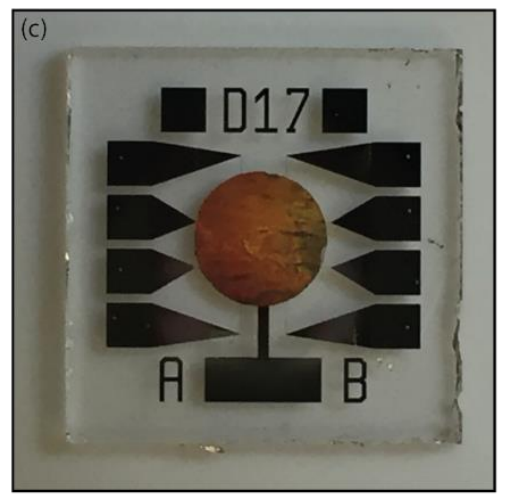

Figure S1. Schematic and photographs of microfabricated $3 \omega$ measurement devices. (a) Schematic layout showing different functions of a single die, including two four-point-probe heater/thermometer patterns, a buried working electrode, and the patterned seed layer where the sample is deposited. (b) Photograph of a representative device immediately following microfabrication. (c) Photograph of the same device after depositing a nickel inverse opal with $1000 \mathrm{~nm}$ pore diameter. 
(a)

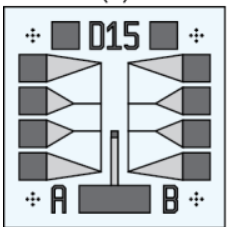

(e)

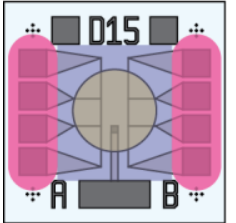

(b)

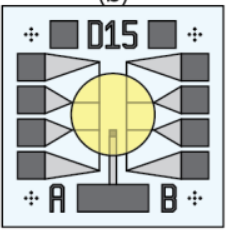

(f)

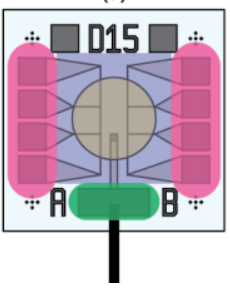

(c)

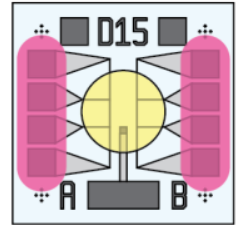

(g)

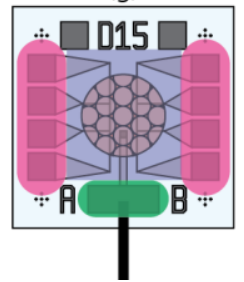

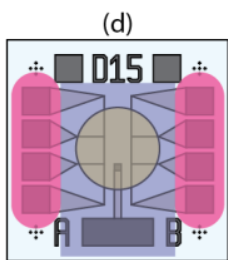

(h)

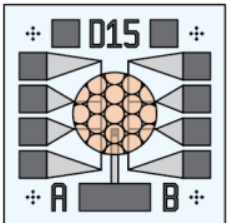

Legend:

Fused Silica Wafer

$\mathrm{SiO}_{2}$ Passivation

Buried Ti/Pt

Exposed Ti/Pt

Lacquer

Opal

Ag Paint + Epoxy

Ti/Au Seed Layer

Inverse Opal

Figure S2. Processing steps for synthesizing patterned inverse opals on microfabricated $3 \omega$ measurement devices. (a) As-synthesized device. (b) Metal seed layer is evaporated through a shadow mask. (c) Fingernail lacquer is applied over the contact pads. (d) The opal is self-assembled on the device. (e) The opal is removed from the working electrode contact pad using a razor blade. (f) A polytetrafluoroethylene-coated wire is attached to the working electrode pad using silver paint, and the junction is covered by dielectric epoxy. (g) The inverse opal is electrodeposited onto the patterned metal seed layer. (h) The substrate is immersed in tetrahydrofuran to simultaneously dissolve the polystyrene spheres, fingernail lacquer, epoxy, and colloidal silver paint.

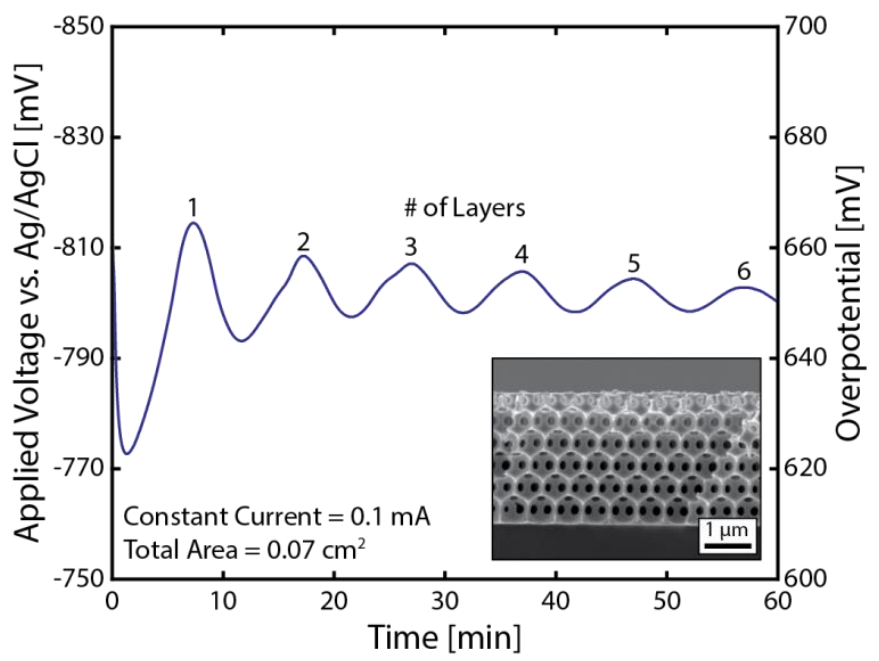

Figure S3. Example galvanostatic electrodeposition characteristic for nickel electrodeposition at a constant current of $100 \mu \mathrm{A}$ over a total seed layer area of $0.07 \mathrm{~cm}^{2}$ (effective current density $\sim 1.5 \mathrm{~mA} / \mathrm{cm}^{2}$ ). For a crystalline opal, the deposition area changes along each layer, and for constant current this causes oscillations in the voltage signal. Each peak corresponds to a single layer of the inverse opal. The inset scanning electron microscopy image shows a cross-section of the corresponding $600 \mathrm{~nm}$-diameter nickel inverse opal sample.

Table S1. Galvanostatic electrodeposition parameters for copper and nickel inverse opals

\begin{tabular}{ccccccc} 
Metal & Electrolyte Composition (aqueous) & $\mathrm{pH}$ & $\begin{array}{c}\mathrm{V}_{\mathrm{OC}} \\
{[\mathrm{mV}]}\end{array}$ & $\begin{array}{c}\text { Current } \\
{[\mathrm{mA}]}\end{array}$ & $\begin{array}{c}\text { Seed Layer } \\
\text { Area }\left[\mathrm{cm}^{2}\right]\end{array}$ & $\begin{array}{c}\text { Effective Current } \\
\text { Density }\left[\mathrm{mA} / \mathrm{cm}^{2}\right]\end{array}$ \\
\hline Copper & $0.6 \mathrm{M} \mathrm{CuSO}_{4}+5 \mathrm{mM} \mathrm{H}_{2} \mathrm{SO}_{4}$ & 2.70 & +80 & 0.5 & 0.07 & 7.5 \\
Nickel & $1 \mathrm{M} \mathrm{NiSO}_{4}+0.2 \mathrm{M} \mathrm{NiCl}_{2}+0.6 \mathrm{M} \mathrm{H}_{3} \mathrm{BO}_{3}$ & 3.58 & -150 & 0.1 & 0.07 & 1.5
\end{tabular}




\section{Section 2. Material Characterization of Inverse Opals}

The quality of the inverse opal crystal can be inferred from its optical properties. For both copper and nickel inverse opals in the present work with pore diameters 300-1000 nm, visible colors can be seen under incident white light. The spatial uniformity of the colors is indicative of uniform metal deposition and monodispersity of the pore size. The copper and nickel inverse opals with $100 \mathrm{~nm}$ pore diameter appear matte and are dark brown and dark gray, respectively.

(a) Blue

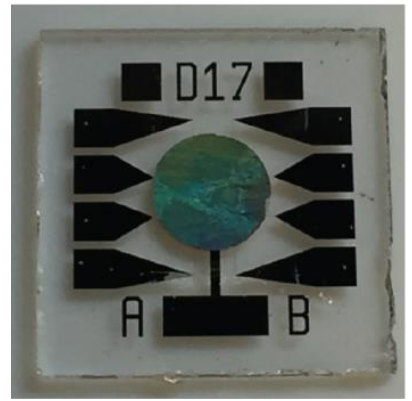

(b) Green

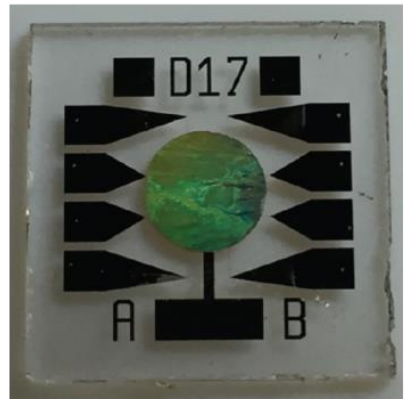

(c) Yellow

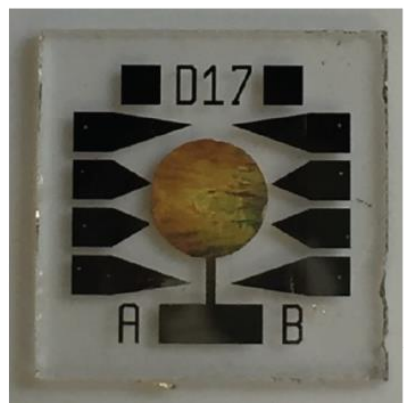

(d) Red

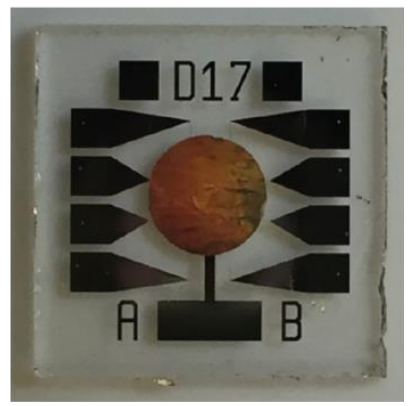

Figure S4. Photographs of a single nickel inverse opal with $1000 \mathrm{~nm}$-diameter pores patterned onto a $3 \omega$ measurement device as viewed from different angles.

The long-range order and crystallinity of an inverse opal can be quantified by taking a twodimensional Fourier transform of a low-magnification scanning electron microscopy image. ${ }^{1}$ The Fourier transform is consistent with a triangular lattice, and line scans along the principal axes show peaks that correspond to the reciprocal lattice spacing of the inverse opal crystal.
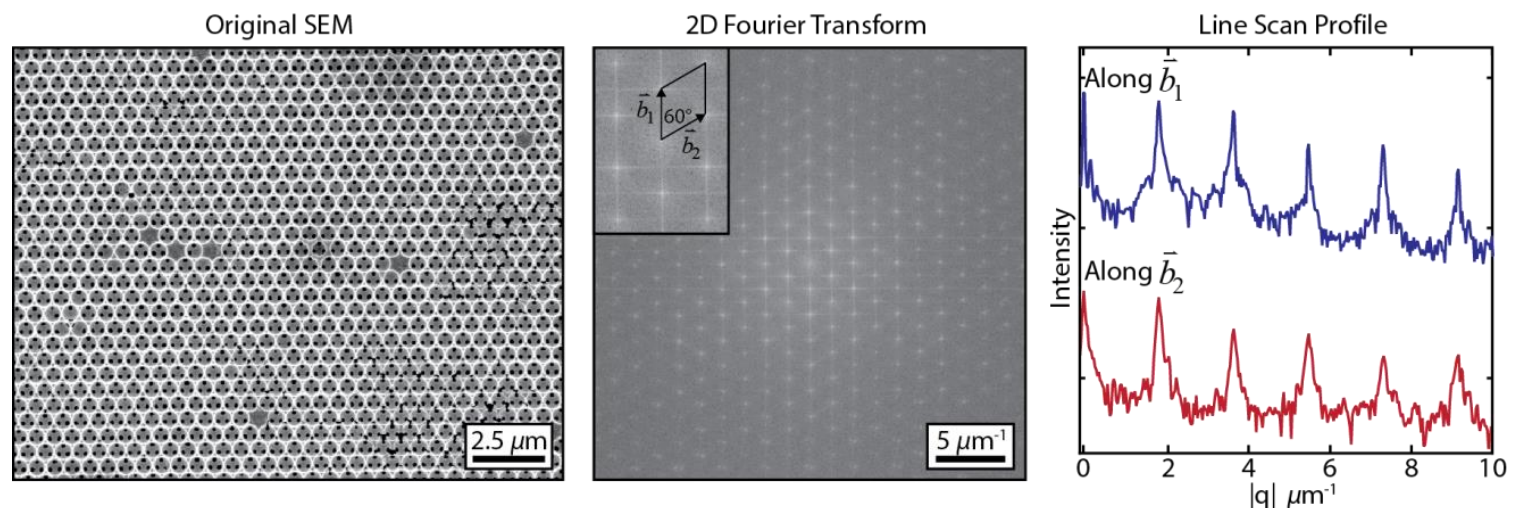

Figure S5. Fourier transform of a nickel inverse opal scanning electron microscopy (SEM) image. The appearance of a diffraction-like pattern demonstrates crystallinity and long-range order in the porous morphology. 
Nickel inverse opals are observed to have both regions of long-range surface uniformity (see Fig. S5) and regions where the top layer of pores has a spatially-varying filling fraction (see Fig. S6), which has been shown in the literature to result in different perceived photonic properties of the crystal. ${ }^{2}$ Copper inverse opals exhibit more surface roughness compared to nickel inverse opals due to less uniform electrodeposition. While the structure of the crystal is similar for both nickel and copper inverse opals, the photonic properties of the copper inverse opals used in the present work are partially suppressed in some regions due to the additional surface roughness.

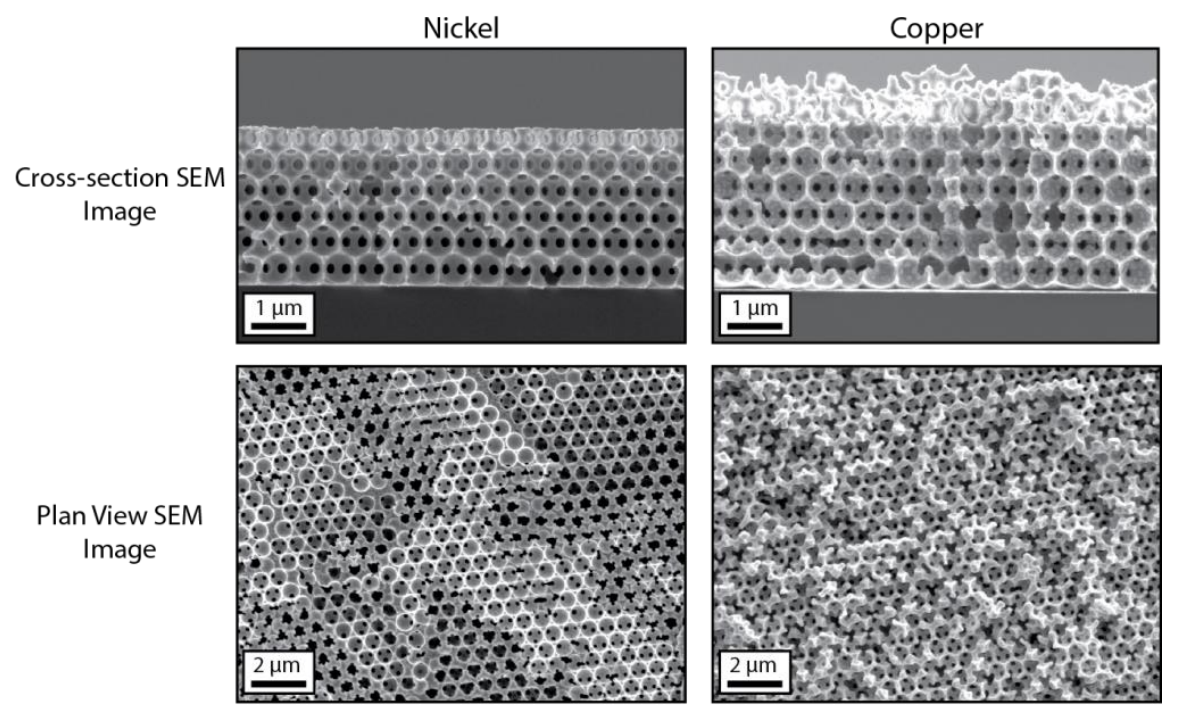

Figure S6. Scanning electron microscopy (SEM) images of a representative nickel inverse opal (left) and a copper inverse opal (right) having a pore diameter of $600 \mathrm{~nm}$. Both of these samples exhibit differing degrees of spatiallyvarying surface nonuniformities and roughness, but this effect can be minimized through optimized electrodeposition conditions.

We use X-ray diffraction (XRD) to estimate the average grain size in the metal and to demonstrate that the grain size is invariant of inverse opal pore size. We use XRD measurements of both copper and nickel inverse opals with pores having the smallest $(d=100 \mathrm{~nm})$ and largest $(d=1000 \mathrm{~nm})$ pores used in the present work. The XRD measurements are performed on the same inverse opals used for the thermal measurements. For small crystal domains (less than a few hundred nanometers), the average grain size $d_{\mathrm{gb}}$ is estimated using the Scherrer equation: ${ }^{3,4}$ 


$$
d_{g b}=\frac{\kappa \lambda}{B \cos \theta}
$$

where $\kappa$ is the crystallite-shape factor, $\lambda$ is the wavelength of the X-rays, $B$ is the full-width of the peak at half-maximum (FWHM), and $\theta$ is the Bragg angle. While Eq. S1 provides an estimate of the absolute average grain size, here we use the FWHM of the characteristic peaks as a proxy for grain size, where changes in the peak width $B$ correspond to changes in $d_{\mathrm{gb}}$. We observe negligible changes in $B$ between the extreme limits of pore size for both copper and nickel (see Fig. S7); this suggests that there is not an appreciable change in grain size across the samples used in the present work. We fit a Gaussian curve to each peak and use Eq. S1 to estimate the average grain size $d_{\mathrm{gb}}$ to be $\sim 24 \mathrm{~nm}$ and $\sim 21 \mathrm{~nm}$ for copper and nickel, respectively.

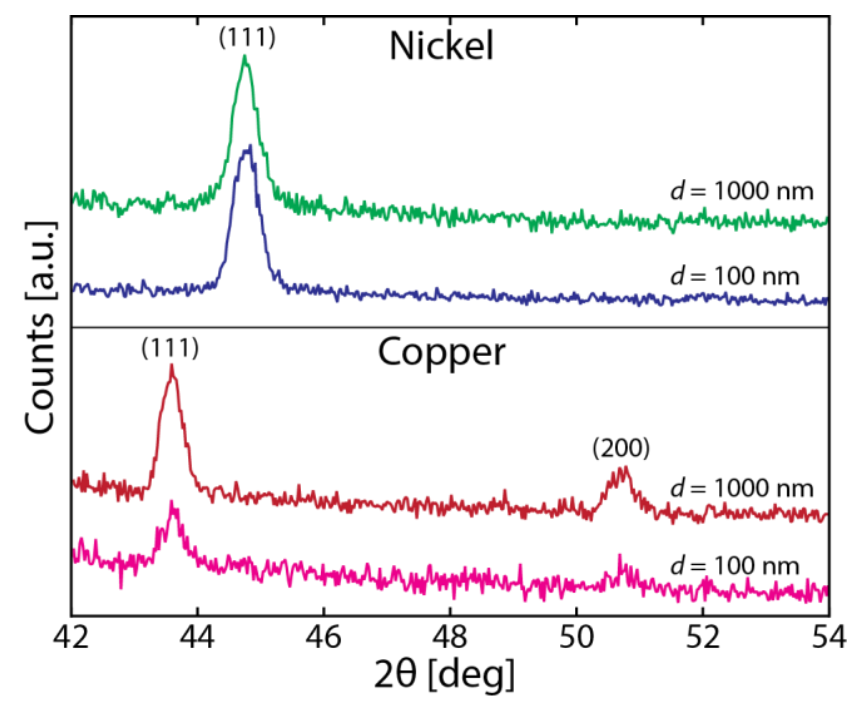

Figure S7. X-ray diffraction (XRD) data for copper and nickel inverse opals having pore diameters of $d=100 \mathrm{~nm}$ and $d=1000 \mathrm{~nm}$. The average grain size of the metal can be inferred from the width of the characteristic peaks, which does not appear to change with the inverse opal pore size.

Both nickel and copper form $\sim 1 \mathrm{~nm}$ of native surface oxide ${ }^{5-7}$ when exposed to ambient conditions, which we have verified using transmission electron microscopy. However, the presence of a surface oxide is not expected to change the nature of the electron surface scattering, which is primarily diffusive when the surface roughness is greater than $\sim 1 \mathrm{~nm} .{ }^{8,9}$ 
Instead, the surface oxide reduces the volume of pure metal in the IO conduction pathway, and the percentage of oxide increases as the IO pore diameter is reduced (due to a larger exposed surface area). Since the conductivity of nickel IOs remains constant with pore size, we expect the role of oxidation in copper IOs to be small relative to the contributions of morphology and size effects. In the limit of a copper IO with $d=100 \mathrm{~nm}$, the maximum reduction in volume is only $16 \%$, which is substantially smaller than the observed $57 \%$ reduction in thermal conductivity. For a copper IO with $d=1000 \mathrm{~nm}$, this volume reduction decreases to less than $2 \%$.

\section{Section 3. 3 $\omega$ Device Characterization, Sensitivity Analysis, and Uncertainty Analysis}

A fused silica wafer is patterned into the configuration shown in Fig. 4a using photolithography and electron beam evaporation of $5 \mathrm{~nm}$ titanium and $60 \mathrm{~nm}$ platinum. The pattern for each die consists of two symmetric four-point-probe heater/thermometer lines for thermal measurements and a single working electrode line for electrodeposition. A passivation film is then deposited onto the surface of the wafer to electrically-isolate the buried metal lines from the conductive inverse opals. This passivation layer includes $40 \mathrm{~nm} \mathrm{SiO}{ }_{2}$ deposited by atomic layer deposition (ALD), $170 \mathrm{~nm} \mathrm{SiO}{ }_{2}$ deposited by high density plasma-enhanced chemical vapor deposition, and an additional $40 \mathrm{~nm} \mathrm{SiO}$ 2 deposited by ALD. The contact pads and a window over the working electrode at the center of the device are exposed using photolithography and buffered oxide etch. Lastly, the wafer is diced into individual square dies (74 dies per 4-inch wafer) that are $9 \mathrm{~mm}$ on each side. The working electrode line consists of a single contact pad at the edge of the die, a buried line that leads to the center of the device, and a small window that exposes the end of the line. This is used during electrodeposition to carry current from the contact pad to the seed layer.

Two calibration steps performed for each device using the $3 \omega$ method with a driving current of $3 \mathrm{~mA}$ over the frequency range 200-3000 Hz. First, the as-fabricated device is 
calibrated to measure the thermal properties of the fused silica wafer and the width of the metal heater line. The sensitivity of each heater line, which is defined by the linear change in resistance with temperature $\mathrm{d} R / \mathrm{d} T$, is independently measured for each device using a vacuum oven. The thermal conductivity and heat capacity of the wafer are measured to be $1.29 \mathrm{~W} \mathrm{~m}^{-1} \mathrm{~K}^{-1}$ and $1.69 \times 10^{6} \mathrm{~J} \mathrm{~m}^{-3} \mathrm{~K}^{-3}$, respectively, and the heater width ranges from 5.1-5.6 $\mu \mathrm{m}$ (nominal width of $5 \mu \mathrm{m})$. After the metal seed layer is deposited, a second calibration is performed to measure the effective thermal conductivity of the seed layer, which is found to range from $90-120 \mathrm{~W} \mathrm{~m}^{-1} \mathrm{~K}^{-1}$. Additional details about the fabrication and characterization of the microfabricated substrates have been previously reported, ${ }^{10}$ and the instrumentation used to isolate the relevant signals during a $3 \omega$ measurement is shown in Fig. S8.

Schematic of $3 \omega$ Measurement

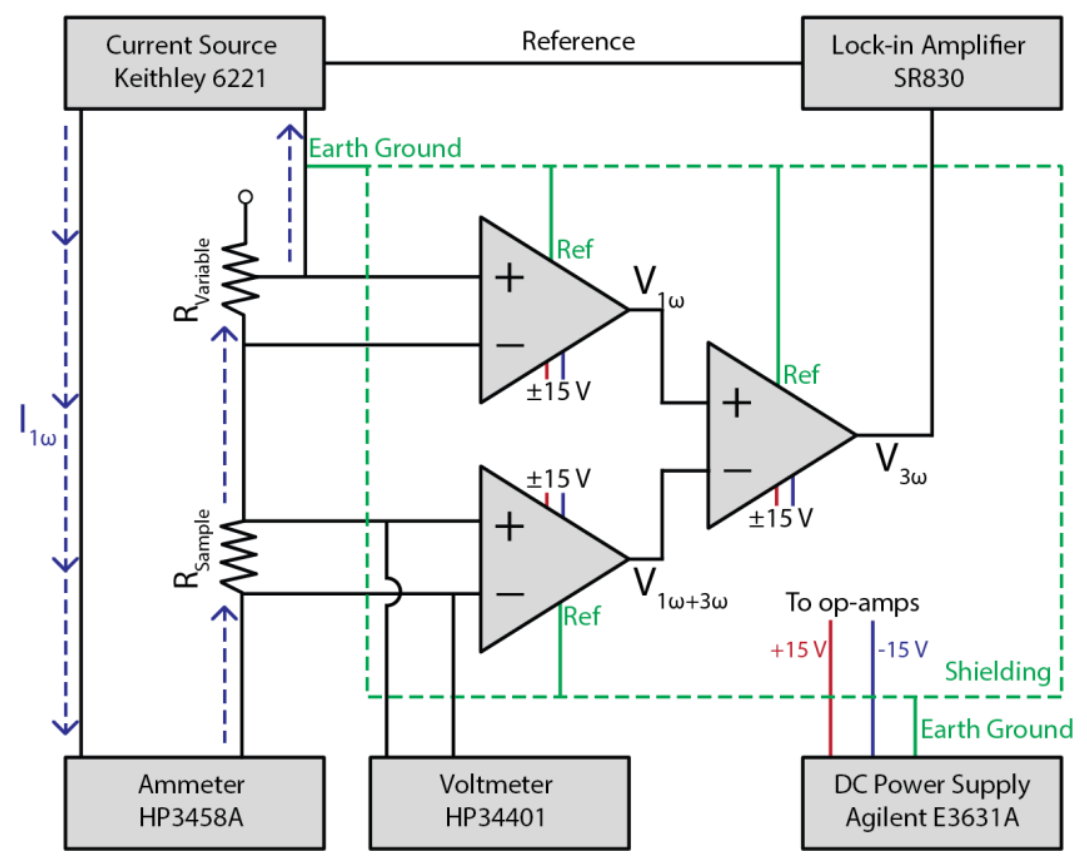

Implementation of AD8221 Op-amps

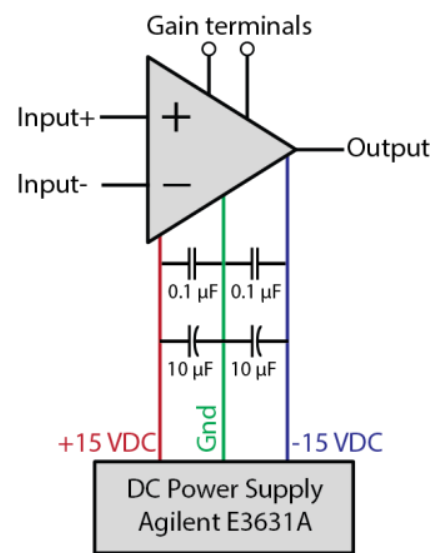

Figure S8. Schematic of electrical signals used in the $3 \omega$ measurement technique. The current and voltage at frequency $1 \omega$ are measured to determine the total heating power along the heater/thermometer line. A variable resistor (with low temperature coefficient of resistance) is set to the same resistance as the sample and placed in series with the sample. Cascaded differential operational amplifiers (Analog Devices AD8221) are then used to minimize the $1 \omega$ signal and to isolate the $3 \omega$ voltage signal, which is measured using a lock-in amplifier. 
The sensitivity of the $3 \omega$ method to different thermophysical properties of the device and sample is quantified by considering the differential change in the measured temperature rise $\Delta T$ caused by a differential perturbation in a specific thermophysical property $X$ about a nominal value. The sensitivity coefficient $S_{X}$ for parameter $X$ is often defined as: ${ }^{10-12}$

$$
S_{X}=\frac{\partial \ln (\Delta T)}{\partial \ln (X)}
$$

For all other parameters fixed, $S_{X}$ indicates the relative sensitivity of a particular $3 \omega$ configuration to parameter $X$. Figure S9 shows that the magnitude and the sign of the sensitivity curves are comparable for both film thickness $L_{\mathrm{IO}}$ and conductivity $k_{\mathrm{eff}}$; this suggests that the measurement configuration is primarily sensitive to the product of these two properties, which corresponds to the in-plane thermal resistance of the inverse opal $R_{\text {in-plane }} \sim\left(k_{\text {eff }} \times L_{\mathrm{IO}}\right)^{-1}$. We therefore require an independent measurement for sample thickness to uniquely extract the inverse opal thermal conductivity.
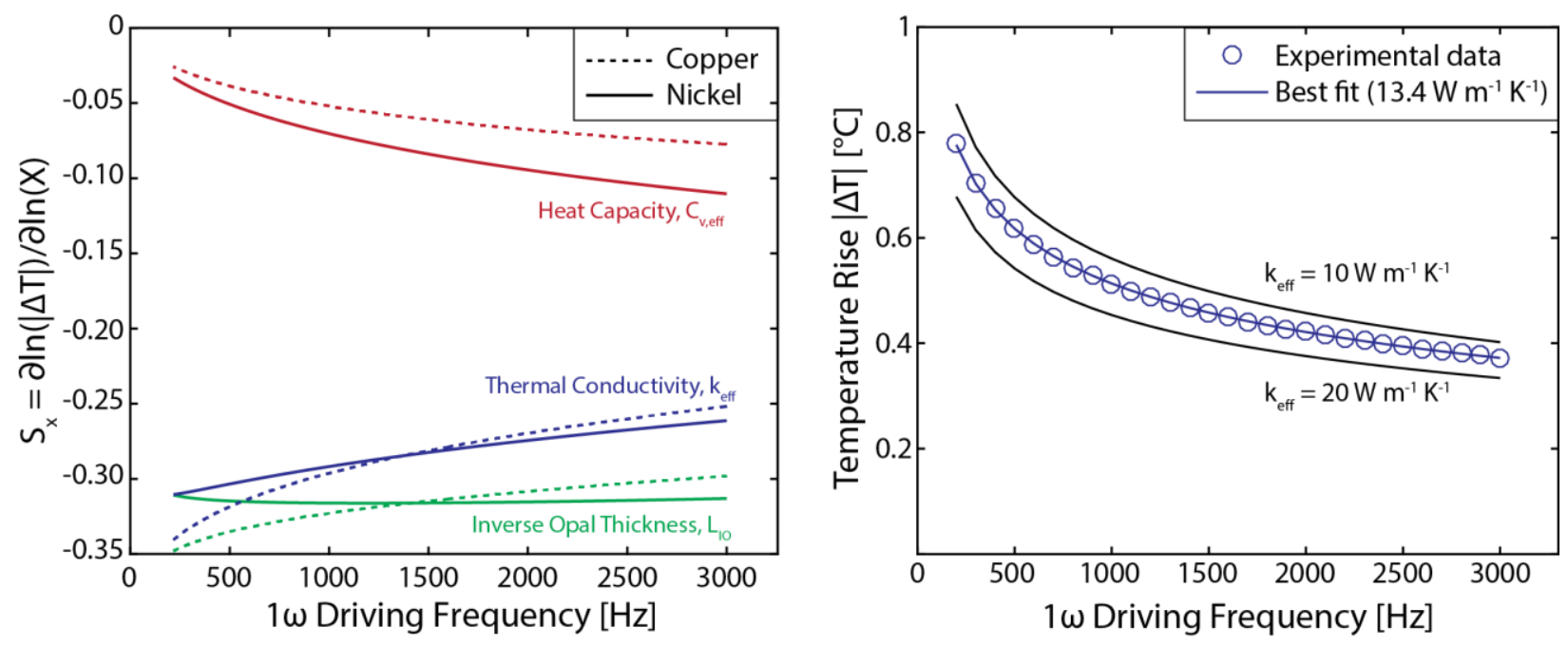

Figure S9. (left) Sensitivity analysis for copper (dashed lines) and nickel (solid lines) inverse opals. (right) Representative data fit for nickel inverse opal using the $3 \omega$ method. The blue circles and solid blue line correspond to the experimental data and the best fit of the thermal properties to the data, respectively. The solid black lines show the expected thermal response for different thermal conductivity values. 
Furthermore, we can validate the assumption of isotropic conduction by performing a simultaneous multiparameter fit for a separate in-plane conductivity $k_{\mathrm{xy}}$ and cross-plane conductivity $k_{\mathrm{z}}$. For the samples in the present work, there is relatively low sensitivity to the cross-plane conductivity $k_{\mathrm{z}}$ since the films are both thermally conductive and thermally thin (relative to the thermal penetration length $L_{\text {thermal }} \approx \sqrt{\alpha / \omega}$, where $\alpha$ is the thermal diffusivity and $\omega$ is the heating frequency). To achieve the best cross-plane sensitivity, we consider the analysis for the sample having the highest cross-plane thermal resistance, which corresponds to the thickest nickel inverse opal. When we assume an isotropic conductivity, we measure $k_{\text {eff }}=k_{\mathrm{xy}}=$ $k_{\mathrm{z}}=16.2 \mathrm{~W} \mathrm{~m}^{-1} \mathrm{~K}^{-1}$ (see Table S2). When we instead fit the same data for separate in-plane and cross-plane conductivities, we measure the in-plane conductivity $k_{\mathrm{xy}}=16.2 \mathrm{~W} \mathrm{~m}^{-1} \mathrm{~K}^{-1}$ and the cross-plane conductivity $k_{\mathrm{z}}=17.2 \mathrm{~W} \mathrm{~m}^{-1} \mathrm{~K}^{-1}$, a difference of only $6 \%$. Since the sensitivity to the cross-plane conductivity is nearly an order of magnitude smaller than the sensitivity to the inplane conductivity, we find this difference to fall within the uncertainty of the thermal conductivity measurement. Consequently, we believe that the isotropic conduction assumption (i.e. $k_{\mathrm{xy}}=k_{\mathrm{z}}=k_{\mathrm{eff}}$ ) is valid, which follows from the expectation that the nominal material architecture is isotropic.

The principal source of uncertainty in the measurement is the film thickness of each inverse opal, which is measured using cross-sectional scanning electron microscopy. This measurement configuration is predominantly sensitive to the lateral thermal resistance of inverse opal, and uncertainty in the film thickness directly results in uncertainty in the thermal conductivity. Copper electrodeposition tends to result in a higher variation in film thickness across a given sample compared to nickel electrodeposition, and this leads to larger uncertainty in thermal conductivity for copper inverse opals compared to nickel inverse opals. There are 
additional sources of propagating uncertainty that are found to be appreciable to the extraction of thermal conductivity data from the measurement signal, and these are primarily comprised of uncertainty in the measured values for heater width, heater sensitivity $\mathrm{d} R / \mathrm{d} T$, and the metal seed layer thermal conductivity. Other parameters, including instrument noise $(\sim 1 \mu \mathrm{V}$ at a lock-in time constant of $100 \mathrm{~ms}$, or a signal-to-noise ratio of $\sim 10^{3}$ ) are found to be negligible with respect to measurement uncertainty. The thermal conductivity data shown in Fig. 5b are tabulated below in Table S2, which includes the thickness of each inverse opal measured in the present work. The thickness is measured using cross-sectional scanning electron microscopy images at a minimum of 10 locations, and the uncertainty corresponds to the standard deviation from all measurements.

Table S2. Tabulated thermal conductivity data for inverse opals used in the present work

\begin{tabular}{lccc} 
Metal & $\begin{array}{c}\text { Pore Diameter } \\
{[\mathrm{nm}]}\end{array}$ & $\begin{array}{c}\text { Thickness } \\
{[\mu \mathrm{m}]}\end{array}$ & $\begin{array}{c}\text { Thermal Conductivity } \\
{\left[\mathrm{W} \mathrm{m}^{-1} \mathrm{~K}^{-1}\right]}\end{array}$ \\
\hline Copper & 100 & $2.76 \pm 0.17$ & $27.1 \pm 1.8$ \\
Copper & 300 & $4.73 \pm 0.20$ & $39.7 \pm 1.8$ \\
Copper & 400 & $3.37 \pm 0.20$ & $45.9 \pm 3.4$ \\
Copper & 600 & $4.71 \pm 0.25$ & $57.1 \pm 3.5$ \\
Copper & 1000 & $6.55 \pm 0.19$ & $62.7 \pm 2.0$ \\
Nickel & 100 & $2.13 \pm 0.10$ & $12.5 \pm 1.4$ \\
Nickel & 300 & $4.41 \pm 0.20$ & $13.4 \pm 0.8$ \\
Nickel & 400 & $3.00 \pm 0.10$ & $16.0 \pm 1.3$ \\
Nickel & 600 & $2.70 \pm 0.10$ & $15.2 \pm 0.7$ \\
Nickel & 1000 & $5.44 \pm 0.11$ & $16.2 \pm 0.4$
\end{tabular}

\section{Section 4. Additional Details of Conduction Model}

The evaluation of the surface scattering integral (Eq. 5) for a complex geometry requires statistical sampling as given by Eq. 6. Each particle $i$ is treated as a discrete sample having an arbitrary point of origin, trajectory, and line-of-sight distance $L_{\mathrm{i}}$ from the origin to the first 
intersection with a surface. After $n$ samples, we generate a statistical distribution and a mean value for $L$ that converges for $10^{5}$ samples (see Fig. S10).
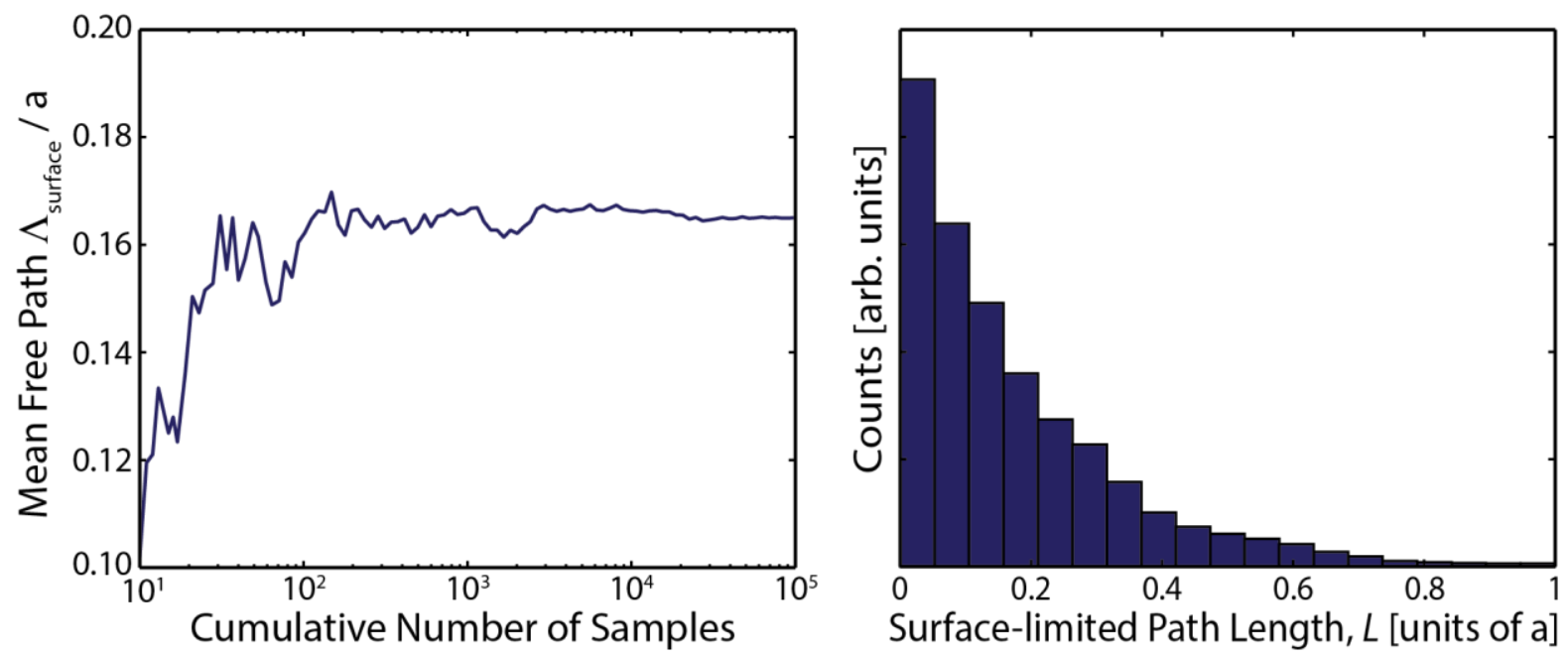

Figure S10. (left) Convergence of surface scattering integral (Eq. 5) as demonstrated by evaluating Eq. 6 as a function of the cumulative number of sampled points. (right) Statistical distribution of all particle travel lengths $L_{\mathrm{i}}$ relative to the FCC inverse opal unit cell size (with side length $a$ ) after $10^{5}$ samples.

The electron mean free path in a bulk metal is estimated from the Drude-Sommerfeld model for electron transport. The mean free path $\Lambda_{\text {diff }}$ can be written in terms of the average electron velocity and the average time between electron scattering events:

$$
\Lambda_{\text {diff }}=v_{F} \tau
$$

where $v_{\mathrm{F}}$ is the Fermi velocity and $\tau$ is the relaxation time. For a bulk metal, both $v_{\mathrm{F}}$ and $\tau$ can be estimated from a combination of fundamental constants, the volumetric free electron density $n$, and the electrical resistivity of the metal $\rho:^{13}$

$$
\begin{gathered}
v_{F}=\left(\frac{\hbar}{m}\right)\left(3 \pi^{2} n\right)^{1 / 3} \\
\tau=\frac{m}{\rho n e^{2}}
\end{gathered}
$$

where $\hbar$ is the reduced Planck's constant and $m$ is the rest mass of an electron. An expression for $\Lambda_{\text {diff }}$ in terms of $\rho$ and $n$ is obtained by combining Eqs. S3-S5: 


$$
\Lambda_{\text {diff }}=\frac{\hbar}{\rho e^{2}}\left(\frac{3 \pi^{2}}{n^{2}}\right)^{1 / 3}
$$

We evaluate $\Lambda_{\text {diff }}$ for copper and nickel using data for the bulk resistivity $\rho$ from the literature ${ }^{14}$ and the free electron density $n$ either (for copper) from the literature ${ }^{13}$ or (for nickel) from the evaluation of:

$$
n=\frac{N_{A} z \rho_{m}}{M M}
$$

where $N_{\mathrm{A}}$ is Avogadro's number, $z$ is the number of valence electrons per atom, $\rho_{\mathrm{m}}$ is the mass density, and $M M$ is the molar mass of the metal. We validate this model by comparing $\Lambda_{\text {diff }}$ computed using Eq. S6 to first-principles calculations for the electron mean free path in Au and Ag presented by Jain and McGaughey. ${ }^{15}$ Since there is a distribution of electron mean free paths in metals, we estimate $\Lambda$ to be the mean free path corresponding to where the thermal conductivity accumulation function reaches $50 \%$ of the total thermal conductivity. For Au and $\mathrm{Ag}$, the evaluation of Eq. S6 deviates from the first-principles calculations by only 6 and $3 \mathrm{~nm}$, respectively. Therefore, we expect Eq. S6 to give a reasonable prediction for the electron mean free path in other similar metals, including nickel and copper used in the present work. These calculated results are tabulated in Table S3. A more comprehensive tabulation of calculated electronic properties of metals, including mean free path, is compiled by Gall. ${ }^{16}$

Table S3. Estimated values of the electron mean free path $\Lambda$ near room temperature.

\begin{tabular}{cccccc} 
Metal & $\begin{array}{c}\text { Free Electron } \\
\text { Density } n\left[\mathrm{~cm}^{-3}\right]\end{array}$ & $\begin{array}{c}\text { Resistivity } \rho \\
{[\mu \Omega-\mathrm{cm}]}\end{array}$ & $\begin{array}{c}\text { Mean Free Path } \\
(\text { Eq. S6) } \Lambda[\mathrm{nm}]\end{array}$ & $\begin{array}{c}\text { First-principles }{ }^{15} \\
\Lambda[\mathrm{nm}]\end{array}$ & $\begin{array}{c}\text { First-principles }^{16} \\
\Lambda[\mathrm{nm}]\end{array}$ \\
\hline Copper & $8.47 \times 10^{22}$ & 1.696 & 39 & & 40 \\
Nickel & $18.3 \times 10^{22}$ & 7.028 & 6 & & 6 \\
Gold & $5.90 \times 10^{22}$ & 2.256 & 37 & 31 & 38 \\
Silver & $5.86 \times 10^{22}$ & 1.677 & 50 & 47 & 53
\end{tabular}




\section{Section 5. Electrical Conductivity Measurement}

The electrical resistance of an inverse opal is measured by patterning the metal seed layer $(5 \mathrm{~nm}$ titanium and $50 \mathrm{~nm}$ gold) into a four-point-probe configuration on a dielectric surface as shown in Fig. S11. We deposit the metal seed layer using electron beam evaporation through a shadow mask onto a thermally-oxidized silicon wafer. We use a high aspect ratio line to increase the total resistance of the sample and to improve measurement sensitivity compared to a standard sheet resistance measurement. The resistance measurement is performed using an I-V sweep and performing a linear regression. First, we measure the electrical resistance of the metal seed layer, which is found to be $\sim 30 \Omega$. Next we deposit the inverse opal over the line and measure the composite resistance, which includes parallel contributions from the seed layer and the inverse opal. Since the inverse opal is comparatively thick $(\sim 3 \mu \mathrm{m})$, the measured composite resistance is reduced to $\sim 1 \Omega$. The inverse opal length $L_{\mathrm{IO}}$, width $w_{\mathrm{IO}}$, and thickness $t_{\mathrm{IO}}$ are all measured using scanning electron microscopy. By subtracting out the seed layer contribution and measuring the exact geometric dimensions of the inverse opal, we can directly calculate the electrical resistance of the inverse opal $R_{\mathrm{IO}}$ and consequently the electrical conductivity $\sigma$ :

$$
\sigma=\frac{L_{I O}}{R_{I O}\left(w_{I O} t_{I O}\right)} .
$$

Future electrical conductivity measurements would benefit from using photolithographicallypatterned features, which greatly increases the precision of the dimensions and reduces the variation in width along the line. This would also enable higher aspect ratio measurement lines to increase the total resistance of the sample and the overall sensitivity. 


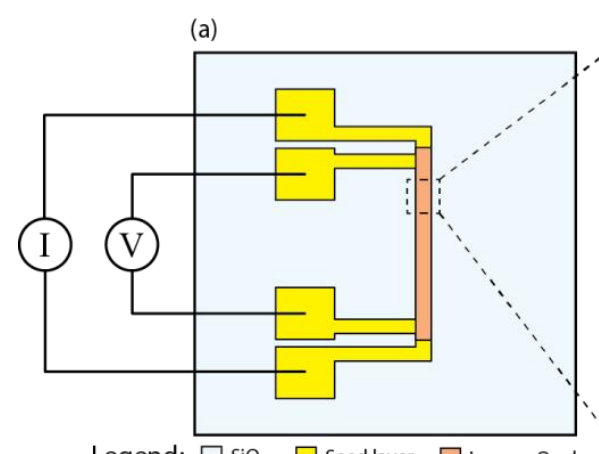

(b)

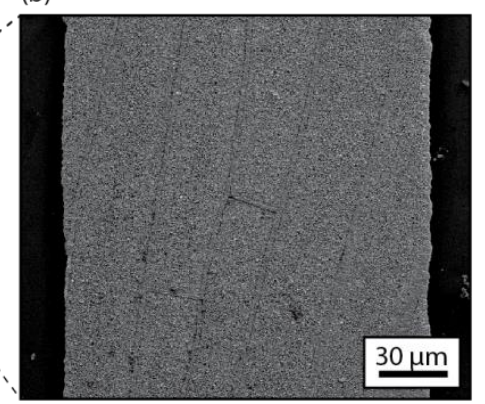

(c)

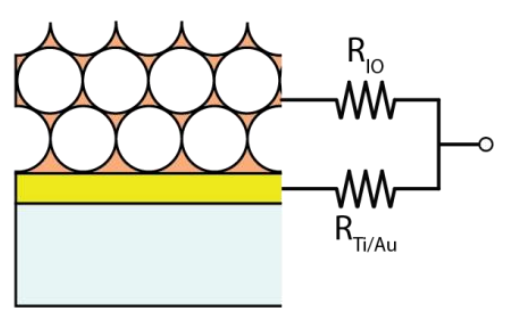

Figure S11. Electrical conductivity measurement of inverse opal. (a) A silicon wafer with a thermally-grown $\mathrm{SiO}_{2}$ surface is used as the substrate, and a four-point-probe pattern with a high aspect ratio line is deposited using electron beam evaporation ( $5 \mathrm{~nm}$ titanium and $50 \mathrm{~nm}$ gold) through a shadow mask. The inverse opal is then electrochemically deposited along the measurement line. (b) Scanning electron microscopy image of a segment of the measurement line that has been covered by a copper inverse opal. (c) The measured electrical resistance is comprised of two parallel resistance contributions: the metal seed layer and the inverse opal.

\section{Section 6. Tradeoff between Surface Area-to-Volume Ratio and Thermal Conductivity}

For FCC copper inverse opals having submicron pore sizes and at room temperature, there is a tradeoff between the effective thermal and electrical conductivity and the surface area-to-volume ratio as shown in Fig. S12. The effective conductivity is evaluated from Eq. 9 for copper and the surface area-to-volume ratio is evaluated from Eq. 10. 


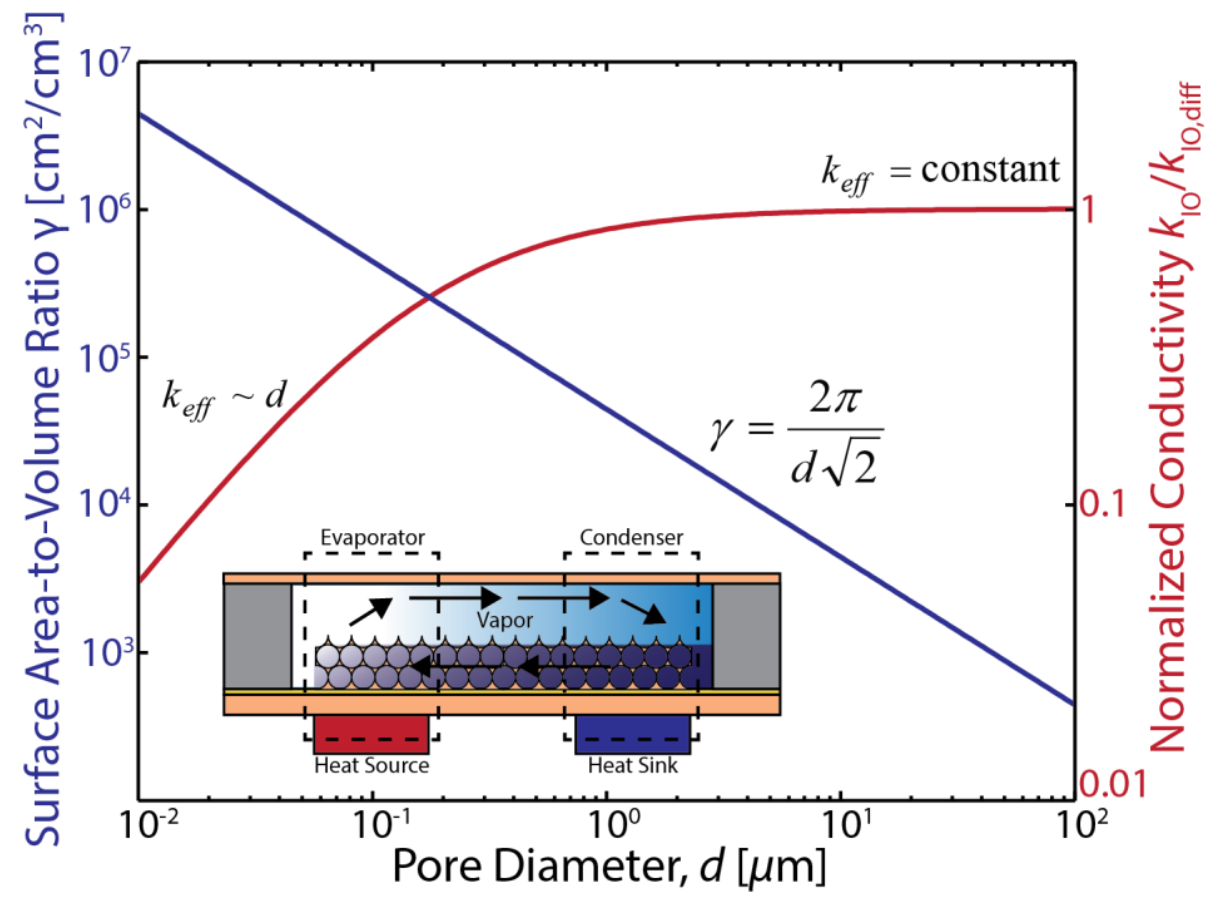

Figure S12. Surface area-to-volume ratio and thermal and electrical conductivity scaling with pore diameter for a close-packed (solid volume fraction $\Phi_{\mathrm{IO}}=0.26$ ) face-centered cubic copper inverse opal. The inset schematic shows an example application of an inverse opal being utilized as the wicking layer in a vapor chamber.

\section{Section 7. Supporting Information References}

(1) Eagleton, T. S.; Searson, P. C. Chem. Mater. 2004, 16, 5027-32.

(2) Yu, X.; Lee, Y. J.; Furstenberg, R.; White, J. O.; Braun, P. V. Adv. Mater. 2007, 19, (13) 1689-92.

(3) Scherrer, P. Nachrichten von der Gesellschaft der Wissenschaften zu Göttingen, mathematisch-physikalische Klasse 1918, 1918, 98-100.

(4) Holzwarth, U.; Gibson, N. Nat. Nanotechnol. 2011, 6, (9), 534-534.

(5) Keil, P.; Lützenkirchen-Hecht, D.; Frahm, R., AIP Conference Proceedings, 2007, 882, 490-492.

(6) Suzuki, S.; Ishikawa, Y.; Isshiki, M.; Waseda, Y. Mater. Trans., JIM 1997, 38, (11), 1004-1009.

(7) MacDougall, B., J. Electrochem. Soc., 1983, 130, (1), 114-117.

(8) Feldman, B.; Deng, R.; Dunham, S. T. J. Appl. Phys. 2008, 103, (11), 113715.

(9) Purswani, J. M.; Gall, D. Thin Solid Films 2007, 516, (2-4), 465-469.

(10) Barako, M. T.; Roy-Panzer, S.; English, T. S.; Kodama, T.; Asheghi, M.; Kenny, T. W.; Goodson, K. E. ACS Appl. Mater. Interfaces 2015, 7, (34), 19251-59.

(11) Bauer, M. L.; Norris, P. M. Rev. Sci. Instrum. 2014, 85, (6), 064903.

(12) Roy-Panzer, S.; Kodama, T.; Lingamneni, S.; Panzer, M. A.; Asheghi, M.; Goodson, K. E. Rev. Sci. Instrum. 2015, 86, (2), 024901.

(13) Ashcroft, N. W.; David, M. N., Solid State Physics. Thomson Learning: South Melbourne, 1976.

(14) Kaye, G. W. C.; Laby, T. H., Kaye \& Laby Tables of Physical \& Chemical Constants. National Physical Laboratory: 2005.

(15) Jain, A.; McGaughey, A. J., Phys. Rev. B, 2016, 93, (8), 081206.

(16) Gall, D, J. Appl. Phys., 2016, 119, (8), 085101. 